\title{
Initial gonadotropin levels and sperm parameters differentiate the response to clomiphene citrate in subfertile men
}

\author{
Tommy Jiang^, Vadim Osadchiy, Alvaro Santamaria, Michael H. Zheng, Neilufar Modiri, John T. Sigalos, \\ Keith V. Regets, Jesse N. Mills, Sriram V. Eleswarapu \\ Division of Andrology, Department of Urology, David Geffen School of Medicine, University of California, Los Angeles, CA, USA \\ Contributions: (I) Conception and design: T Jiang, JN Mills, SV Eleswarapu; (II) Administrative support: JT Sigalos, V Osadchiy; (III) Provision of \\ study materials or patients: KV Regets, JN Mills, SV Eleswarapu; (IV) Collection and assembly of data: T Jiang, N Modiri, A Santamaria; (V) Data \\ analysis and interpretation: V Osadchiy, MH Zheng; (VI) Manuscript writing: All authors; (VII) Final approval of manuscript: All authors. \\ Correspondence to: Sriram V. Eleswarapu, MD, PhD. Division of Andrology, Department of Urology, University of California, Los Angeles, 10945 Le \\ Conte Avenue, Ueberroth \#3361, Los Angeles, CA 90095, USA. Email: SEleswarapu@mednet.ucla.edu.
}

Background: Efficacy of clomiphene citrate (CC) in the treatment of male subfertility remains unclear, with inconsistent results in the literature and limited guidance from professional organizations. We sought to stratify the response to clomiphene in men based on their initial gonadotropins and semen parameters.

Methods: We conducted a retrospective analysis of 234 patients from an academic center who took CC for subfertility. Patients with pre-treatment and 3 months follow-up total testosterone (TT) and semen analyses were included. Patients with previous hormone therapy, genitourinary surgery, prior success in conceiving pregnancy, or only one semen analysis were excluded. Primary outcomes were magnitudes of improvement in TT and semen parameters at 3 months. Student's $t$-test (alpha $=0.05$ ) was used for univariate analyses; multivariable linear regression was used for multivariate analysis.

Results: One hundred and thirty-seven patients met inclusion criteria. Thirty-four percent of patients experienced improvement in sperm concentration after 3 months of CC treatment, 13\% decreased, and $53 \%$ showed no change. Using a pre-treatment TT cutoff of $300 \mathrm{ng} / \mathrm{dL}$ and gonadotropin thresholds of $7 \mathrm{miU} / \mathrm{mL}$, initial TT did not affect magnitude of improvement in semen parameters, while lower initial gonadotropins showed statistical improvement across all outcomes. Multivariate analysis showed pretreatment follicle stimulating hormone (FSH) was inversely correlated with improvement in TT [odds ratio (OR): $2.64 \mathrm{e}-05,95 \%$ confidence interval (CI): $1.32 \mathrm{e}-09$ to $5.28 \mathrm{e}-01, \mathrm{P}=0.04]$ and sperm concentration (OR: 0.22 , $95 \%$ CI: $5.70 \mathrm{e}-02$ to $8.48 \mathrm{e}-01, \mathrm{P}=0.03)$. We also provide initial gonadotropin cutoffs that suggest statistical benefit from CC use.

Conclusions: Men with lower gonadotropin levels may expect greater degree of improvement in both hormone and semen parameters with use of CC. Men with azoospermia do not benefit based on semen analyses alone. Degree of non-azoospermia does not affect magnitude of improvement. CC had decreasing efficacy at higher initial gonadotropin levels. These data may provide guidance in stratifying and counseling men for CC treatment.

Keywords: Clomiphene citrate (CC); male subfertility; testosterone; semen parameters

Submitted Nov 08, 2021. Accepted for publication Jan 11, 2022.

doi: 10.21037/tau-21-987

View this article at: https://dx.doi.org/10.21037/tau-21-987

^ ORCID: 0000-0002-1199-0615. 


\section{Introduction}

As many as $15 \%$ of couples will fail to conceive within 1 year of attempted conception, with $50 \%$ of such couples having a male subfertility component (1). Treatments range from lifestyle counseling to surgical procedures that involve manual extraction of sperm (2). Few medical therapies exist for male factor subfertility; these limited therapies are not well studied. Clomiphene citrate (CC) is a selective estrogen reuptake modulator (SERM) that is often empirically prescribed to hypogonadotropic men to improve total testosterone (TT) and semen parameters (3) through the effects of increasing luteinizing hormone (LH) and follicle stimulating hormone (FSH). Previous surveys have shown empiric medical therapy is commonly prescribed among doctors in the context of male factor subfertility (4). Despite the frequent use of these medications, there is limited guidance on the use of these treatment modalities in the American Urologic Association (AUA), European Association of Urology (EAU) and American Society of Reproductive Medicine (ASRM) guidelines $(5,6)$ which only recommends physicians to prescribe SERMs in patients with low testosterone for male infertility.

Data supporting CC for male factor subfertility varies widely. The World Health Organization (WHO), which performed the largest randomized, controlled trial, did not show any improvement in pregnancy rates or sperm concentration with the use of CC compared to placebo (7). However, smaller retrospective and prospective studies with stricter inclusion criteria have noted improvements across varying semen parameters and TT $(8,9)$. The lack of guideline specifications, limited high quality peer review data, and known interprovider variability for the prescription of medications in fertility (10) also raise the question of which men would benefit most from CC. Studies on CC have also typically compared pre- versus post-CC outcomes, but the magnitude of improvement across varying baseline characteristics remains unexplored.

To illustrate which patients may experience the most benefit from CC, we sought to study how differing baseline characteristics (TT, LH, FSH, and sperm concentration) affect fertility related outcomes. We conducted a retrospective analysis of patients seeking family planning who were prescribed CC. Our primary outcomes were magnitude of improvement in TT and semen parameters (semen volume, sperm concentration, total sperm count, and total motile sperm count) after 3-month usage of CC. We hypothesized that oligospermic men with low gonadotropins would experience the greatest benefit. In doing so, we hope to provide granular data on how efficacy of CC varies with baseline gonadotropins. We present the following article in accordance with the STROBE reporting checklist (available at https://tau.amegroups.com/article/ view/10.21037/tau-21-987/rc).

\section{Methods}

\section{Data collection}

We conducted a retrospective analysis of 234 patients who were prescribed CC $[25 \mathrm{mg}$ everyday (QD) or $50 \mathrm{mg}$ every other day (QOD)] for family planning. All patients were extracted from the electronic medical records from a single, tertiary men's health clinic from 6/2015 to 8/2020. We collected the following data: demographic information [age and body mass index (BMI)], pre-CC hormone profiles ( $\mathrm{LH}$, FSH, TT), pre-treatment semen analysis (ejaculate volume, sperm concentration, total sperm count, and total motile sperm count), 3-month follow up post-CC hormone profiles and semen analysis, reproductive assistance history (empiric medical therapies and reproductive assisted procedures), and if pregnancy was eventually achieved via $\mathrm{CC}$ without reproductive assistance or $\mathrm{CC}$ with reproductive assistance. Patients who had prior genitourinary surgeries, prior hormone therapies, were not compliant with CC, or only who only had one semen analysis were excluded from the study. Primary outcomes were magnitude of improvement of TT, semen volume, sperm concentration, total sperm count, and total motile sperm count. The study was conducted in accordance with the Declaration of Helsinki (as revised in 2013). The study was approved by institutional review board of the University of California, Los Angeles (IRB \#20-000710). Prospective consenting of patients was exempt as this was a retrospective study.

\section{Gonadotropin and efficacy of CC analysis}

Patients were stratified by pre-treatment hormone labs $(\mathrm{TT} \geq 300 \mathrm{ng} / \mathrm{dL}$ vs. TT $<300 \mathrm{ng} / \mathrm{dL}, \mathrm{LH}$ $\geq 7 \mathrm{miU} / \mathrm{mL}$ vs. $\mathrm{LH}<7 \mathrm{miU} / \mathrm{mL}, \mathrm{FSH} \geq 7 \mathrm{miU} / \mathrm{mL}$ vs. FSH $<7 \mathrm{miU} / \mathrm{mL}$ ) and also by initial sperm concentration (azoospermia, severely oligozoospermia, moderately oligozoospermia, mildly oligozoospermia, and normospermia) using AUA/ASRM guidelines and WHO/ 
Table 1 Changes in TT and semen parameters stratified by baseline characteristics

\begin{tabular}{|c|c|c|c|c|c|c|c|c|c|c|}
\hline $\begin{array}{l}\text { Pre-treatment } \\
\text { stratification }\end{array}$ & $\begin{array}{l}\text { t Change in } \\
T T \text { (ng/dL) }\end{array}$ & $\mathrm{P}$ & $\begin{array}{c}\text { Change in } \\
\text { semen volume } \\
(\mathrm{mL})\end{array}$ & $P$ & $\begin{array}{l}\text { Change in sperm } \\
\text { concentration } \\
\text { (million } / \mathrm{mL})\end{array}$ & $\mathrm{P}$ & $\begin{array}{l}\text { Change in total } \\
\text { sperm count } \\
\text { (million) }\end{array}$ & $\mathrm{P}$ & $\begin{array}{l}\text { Change in total } \\
\text { motile sperm } \\
\text { count (million) }\end{array}$ & $\mathrm{P}$ \\
\hline $\mathrm{T}<300$ & $285.0 \pm 236.2$ & & $0.0 \pm 1.2$ & & $17.3 \pm 48.8$ & & $29.7 \pm 134.1$ & & $14.2 \pm 81.4$ & \\
\hline $\mathrm{LH} \geq 7$ & $122.9 \pm 228.1$ & 0.13 & $-0.01 \pm 0.99$ & 0.72 & $6.4 \pm 24.8$ & 0.11 & $10.8 \pm 41.1$ & $0.02^{\star}$ & $4.7 \pm 17.7$ & $0.04^{*}$ \\
\hline $\mathrm{FSH} \geq 7$ & $106.9 \pm 244.8$ & $0.03^{*}$ & $0.0 \pm 1.2$ & 0.68 & $1.7 \pm 10.5$ & $<0.01^{\star}$ & $10.9 \pm 30.8$ & $0.01^{*}$ & $6.7 \pm 24.9$ & $0.09^{\star}$ \\
\hline $\mathrm{FSH}<7$ & $227.1 \pm 281.5$ & & $0.1 \pm 1.1$ & & $19.9 \pm 45.1$ & & $49.2 \pm 132.6$ & & $20.2 \pm 67.7$ & \\
\hline Azoospermia & $81.3 \pm 336.0$ & 0.24 & $-0.4 \pm 1.4$ & 0.26 & $1.4 \pm 4.2$ & $<0.01^{*}$ & $2.1 \pm 5.0$ & $<0.01^{*}$ & $0.5 \pm 1.4$ & $<0.01^{\star}$ \\
\hline Non-azo & $205.3 \pm 270.4$ & & $0.1 \pm 1.1$ & & $17.3 \pm 42.1$ & & $43.4 \pm 120.5$ & & $18.3 \pm 61.8$ & \\
\hline
\end{tabular}

Men were stratified by baseline TT, LH, FSH, and semen concentration. Magnitude of improvement was compared between these stratifications. Student's $t$-test was conducted with alpha $=0.05$. ${ }^{*}$, denotes statistical significance (alpha $\left.<0.05\right)$. $T$, total testosterone; $T$, testosterone; $\mathrm{LH}$, luteinizing hormone; $\mathrm{FSH}$, follicle stimulating hormone.

EAU $(11,12)$ criteria, respectively. Gonadotropin cutoffs were chosen arbitrarily as there is limited literature on cutoffs for the usage of CC. Improvements in testosterone and semen analysis parameters were calculated by subtracting the initial value from the post-CC 3-month value. These data were used to generate Table 1 .

\section{Threshold analysis}

We sought to determine the highest $\mathrm{LH}$ and FSH for which patients experience benefit from CC. Using Python version 3.7.0 (Python Inc, Wilmington, Delaware, USA), we systemically performed two-tailed Student's $t$-tests to compare baseline TT and sperm parameters to 3-month post-CC TT and sperm parameters for every gonadotropin value between 0 and $25 \mathrm{miU} / \mathrm{mL}$ (maximum gonadotropin level in our patient population). Figures were generated in the matplotlib package. We then used DigitizeIt Version 2.5, software (13) to derive a threshold value, representing the highest gonadotropin level that we would expect a statistically significant $(\mathrm{P}<0.05)$ improvement in the above mentioned outcome measures. Figure 1 is used as a representative curve to illustrate how these points were determined.

\section{Statistical analysis}

Two-tailed Student's $t$-tests with alpha $=0.05$ were performed for the "Gonadotropin and Efficacy of CC
Analysis" and "Threshold Analysis". We also performed a multivariable linear regression to control for age and BMI, which may represent potential confounders. Age was treated as a continuous variable, BMI as a categorical variable with participants stratified as overweight $(\mathrm{BMI} \geq 25)$ or not overweight $(\mathrm{BMI}<25)$. Due to the high degree of collinearity between LH and FSH levels, we used only FSH in our analysis; however, results were similar regardless of which gonadotropin was used. RStudio v. 1.1.463 (RStudio, Inc., Boston, MA, USA) was used for statistical analysis, with alpha $<0.05$ considered statistically significant.

\section{Results}

One hundred and thirty-seven patients met the inclusion criteria for this study. One hundred and fifteen patients had at least two sets of $\mathrm{LH}, \mathrm{FSH}$, and TT readings: pre-CC and 3-month follow up CC usage. Median follow-up to second semen analysis was 3.8 months (range, 1.2-5.7 months). Patient demographics are illustrated in Table 2.

\section{Predetermined cutoffs}

We found 34\% (46/137) improved sperm concentration categorizations, $13 \%(18 / 137)$ worsened, and 53\% (73/137) did not change. Twenty-three percent (3/13) of azoospermic patients recovered sperm in the ejaculate (two patients with few sperm seen on centrifuged sample, one patient with 


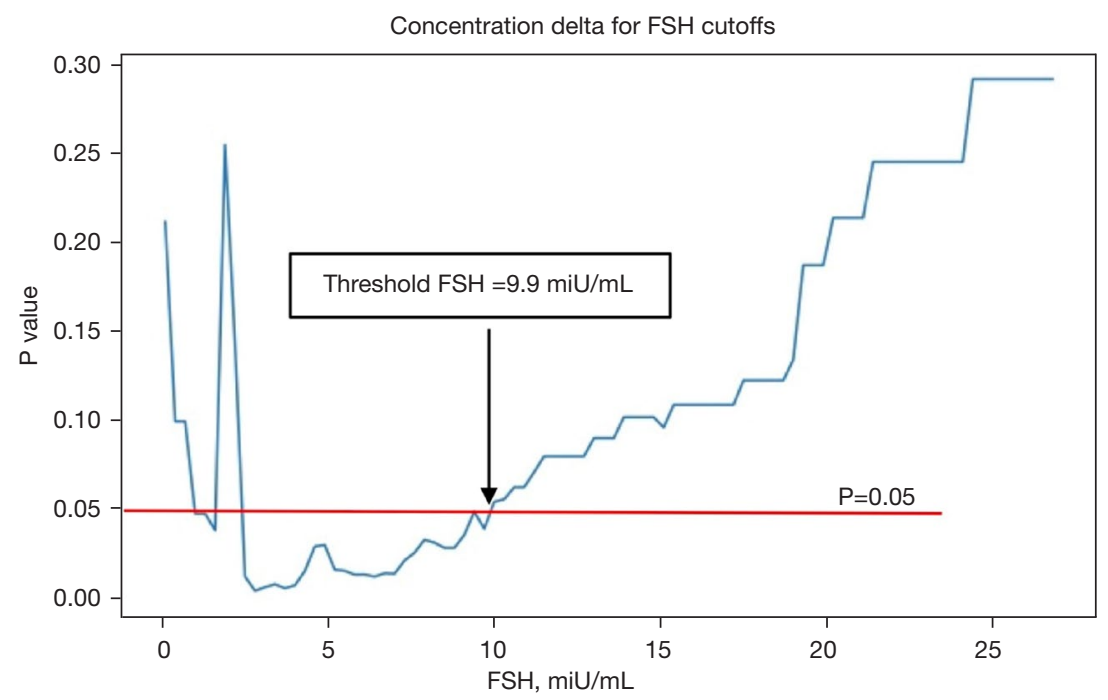

Figure 1 Representative FSH threshold curve for effect of clomiphene citrate on sperm concentration. In this example, the threshold is FSH under which statistically significant differences in sperm concentration can be seen after treatment with clomiphene for 3 months. Y-axis denotes P values; X-axis denotes FSH concentrations. Similar curves were computed for LH and FSH to determine thresholds for improvement in serum testosterone, semen volume, sperm concentration, total sperm count, and total motile sperm count. FSH, follicle stimulating hormone; LH, luteinizing hormone.

Table 2 Patient demographics

\begin{tabular}{lc}
\hline Baseline characteristic & All patients (SD), $\mathrm{n}=137$ \\
\hline Age & $37.7(6.7)$ \\
BMI & $27.7(4.9)$ \\
Pre-treatment LH & $5.1(2.6)$ \\
Pre-treatment FSH & $6(5.2)$ \\
Pre-treatment testosterone & $172.7(152.3)$ \\
Pre-treatment sperm concentration & $25.9(44.8)$ \\
Pre-treatment total sperm count & $45.0(66.1)$
\end{tabular}

$\mathrm{SD}$, standard deviation; BMI, body mass index; $\mathrm{LH}$, luteinizing hormone; $\mathrm{FSH}$, follicle stimulating hormone.

approximately 100,000 sperm/mL).

We found no significant changes in improvement across all semen parameters when stratifying by pre-treatment TT (TT $\geq 300 \mathrm{ng} / \mathrm{dL}$ vs. TT $<300 \mathrm{ng} / \mathrm{dL}$ ). This did not change when using $350 \mathrm{ng} / \mathrm{dL}$ as the TT cutoff. When stratifying by pre-treatment $\mathrm{LH}(\mathrm{LH} \geq 7$ vs. $\mathrm{LH}<7) \mathrm{miU} / \mathrm{mL}$, we found a significant improvement in total sperm count (10.8 ${ }^{4} 41.1$ vs. $\left.45.1 \pm 125.6 \mathrm{M} / \mathrm{mL}, \mathrm{P}=0.02\right)$ and total motile sperm count $(19.1 \pm 64.5$ vs. $4.7 \pm 17.7 \mathrm{M}, \mathrm{P}=0.04)$ favoring the $\mathrm{LH}<7 \mathrm{miU} / \mathrm{mL}$ cohort. We did not observe significant improvement in changes in TT and in sperm concentration when using $\mathrm{LH}$ as a categorical variable (i.e., $\mathrm{LH} \geq 7 v$ s. $\mathrm{LH}$ $<7)$. When stratifying by FSH as a categorical variable (i.e., FSH $\geq 7$ vs. FSH $<7$ ), we found significant improvement in changes in TT $(106.9 \pm 244.8 v s .227 .1 \pm 281.5, \mathrm{P}=0.03) \mathrm{ng} / \mathrm{dL}$, change in sperm concentration $(1.7 \pm 10.5$ vs. $19.9 \pm 34.1 \mathrm{M}, \mathrm{P}<0.01)$, and change in total sperm count $(10.9 \pm 30.8$ vs. $49.2 \pm 132.6 \mathrm{M}$, $\mathrm{P}=0.01)$ favoring the $\mathrm{FSH}<7$ cohort. When stratifying by initial sperm concentration, we found azoospermic patients saw significantly less benefit from CC compared to nonazoospermic patients in all outcomes (Table 1). Upon further analysis, we found degree of non-azoospermia did not alter changes across all outcomes using WHO/EAU criteria. When controlling for age and BMI, we found that lower levels of gonadotropins were similarly associated with improvement in TT (OR: 2.64e-05, 95\% CI: $1.32 \mathrm{e}-09$ to 5.28e-01, $\mathrm{P}=0.04)$, and sperm concentration (OR: 0.22 , $95 \%$ CI: $5.70 \mathrm{e}-02$ to $8.48 \mathrm{e}-01, \mathrm{P}=0.03)$. No statistically significant relationship emerged between gonadotropins and improvement in semen volume, total sperm count, or improvement in motile sperm count.

We found the highest $\mathrm{LH}$ and FSH for which patients still experience benefit from CC for TT was 8.3 and $7.9 \mathrm{miU} / \mathrm{mL}$ respectively. The $\mathrm{LH}$ and $\mathrm{FSH}$ level for change in sperm concentration were 6.2 and $9.9 \mathrm{miU} / \mathrm{mL}$. 
Table 3 Threshold values of LH or FSH to achieve statistically significant increases in serum testosterone, semen volume, sperm concentration, total sperm count, and total motile sperm count

\begin{tabular}{lcc}
\hline Threshold characteristic & $\begin{array}{c}\text { Threshold LH } \\
(\mathrm{miU} / \mathrm{mL})\end{array}$ & $\begin{array}{c}\text { Threshold FSH } \\
(\mathrm{miU} / \mathrm{mL})\end{array}$ \\
\hline Increase in serum testosterone & $\leq 8.3$ & $\leq 7.9$ \\
Increase in semen volume & $\leq 4.8$ & $\leq 9.4$ \\
$\begin{array}{l}\text { Increase in sperm } \\
\text { concentration }\end{array}$ & $\leq 6.2$ & $\leq 9.9$ \\
$\begin{array}{l}\text { Increase in total sperm count } \\
\text { Increase in total motile sperm }\end{array}$ & $\leq 6.3$ & $\leq 8.9$ \\
count & $\leq 6.0$ & $\leq 5.6$ \\
\hline
\end{tabular}

$\mathrm{LH}$, luteinizing hormone; FSH, follicle stimulating hormone.

An LH of 6.3 and FSH of 8.9 was determined for total sperm count (Table 3). Changes in ejaculate volume and total motile sperm count showed low correlation with LH/ FSH and were not included in this study.

\section{Discussion}

Data on CC efficacy vary depending on the outcome studied. CC has been consistently shown to improve symptoms of hypogonadism (14-16) and TT in men with secondary hypogonadism $(14,17,18)$, with a relatively unremarkable side effect profile $(19,20)$. In general, fertility outcomes have been less intensively studied with the available trials limited by smaller sample sizes. Here, we identify the populations of men that would most benefit from CC treatment with respect to TT, sperm parameters, and fertility outcomes.

Most patients did not improve WHO sperm concentration categorizations after taking CC; patients with non-obstructive azoospermia were least likely to benefit, although we did note that three out of 13 azoospermic patients achieved recovery of sperm in the ejaculate. It was unclear from our analysis whether this response is predictable based on a priori factors, due to the small sample size of the azoospermic subset. Hussein et al. found that $64.3 \%$ of men with non-obstructive azoospermia eventually recovered sperm in the ejaculate with prolonged clomiphene monotherapy, and sperm was retrieved with testicular sperm extraction in the other $35.7 \%$ of men (21). Other investigators have shown that optimizing TT in non-obstructive azoospermia using hormone therapy can improve the likelihood of finding sperm during microdissection testicular sperm extraction (mTESE) (22). Our data provide further context that may be helpful in managing expectations in men with azoospermia who wish to explore hormone treatment such as CC. Of note, we found that the degree of non-azoospermia did not affect the magnitude of improvement in sperm parameters when classifying as oligozoospermic or normozoospermic. These data suggest that non-azoospermic patients can experience similar magnitude of improvement when taking CC. It has been previously shown that oligozoospermic men experience benefit in sperm concentration and TT when prescribed CC (8). However, studies have not evaluated CC efficacy when comparing different baseline sperm concentrations.

We identified an inverse relationship between baseline gonadotropins and improvement in TT and sperm parameters. More specifically, results of our multivariate analysis revealed that pre-treatment FSH inversely correlated with changes in TT and sperm concentration. CC improves TT and semen parameters through central modulation of LH and FSH (23); however, previous smaller studies have not found baseline gonadotropins to be predictors of improvement in these outcomes $(24,25)$. In accordance with the mechanism of action of CC, Hashimoto et al. showed significant increases in $\mathrm{LH}$ and FSH upon CC administration in normal men (26). To date, no study has examined the relative contribution of initial gonadotropin levels to degree of improvement in TT or sperm parameters with CC treatment. Our study adds to the existing literature by exploring the benefit of CC by comparing improvements in TT and semen parameters with differing initial gonadotropins.

To better understand the effect on CC and our outcome variables, we stratified our data by gonadotropin levels, identifying the maximum gonadotropin level where patients still experience a benefit to CC therapy. For TT, we found an LH of 8.3 and FSH of 7.9 while for sperm concentration we found an LH of 6.2 and FSH of 9.9. Differences in the LH and FSH cutoffs for these outcomes can be explained in part by $\mathrm{LH}$ playing a more direct role in TT while FSH in sperm maturation and overall testicular health. Additionally, using a gonadotropin cutoff of 7 did not yield statistically significant events for all outcomes, suggesting this number could be optimized. Several studies suggest better guidelines need to be established for CC as many men experience paradoxical worsening semen parameters with CC $(27,28)$. While the general sentiment is CC should be used for hypogonadotropic men, a precise $\mathrm{LH}$ and FSH 
cutoff has not been established. Additionally, intra-provider variability for empiric medical treatment (10) leads to a lack of consensus among fertility specialists of determining the most suitable patients. These different factors could, in part, play a role in the inconclusive reported data of CC on fertility outcomes. Our study provides data that can highlight where patients can still experience benefit from CC based on baseline gonadotropins.

Our study is not without limitations. To reflect the broad prescription of $\mathrm{CC}$, we did not exclude patients based on baseline TT/LH/FSH and initial semen parameters. Secondly, our maximum gonadotropin analysis has not been previously validated and was an observational component of this study. However, since a cutoff has yet to be determined for CC this could serve as a systematic analysis to see which patients still exhibit a statistically significant benefit to taking CC. We performed multiple subset analyses that treated gonadotropins as either continuous variables (multivariate analyses) or categorical variables (threshold analysis around an arbitrary cutoff of $>7$ or $<7 \mathrm{miU} / \mathrm{mL}$ ). Furthermore, a true validation cohort will be needed to confirm these analyses. Lastly, this was a retrospective chart review and was not powered to any given outcome. Additionally, we were limited by the inherent wide variability in semen analysis results and therefore we observed wide variations in outcomes. A true randomized, prospective clinical trial with statistical powering is still needed in this area of study. However, our data highlight interesting characteristics of CC in the context of male subfertility using patient level data.

\section{Conclusions}

Our study highlights not all men benefit equally from CC. Stratifying by differing baseline characteristics, our results can more clearly illustrate the degree of improvement men can expect after CC treatment. The results underscore men with lower gonadotropins experience the most benefit with decreasing benefit with increasing baseline gonadotropin levels. Additionally, non-azoospermic patients experience the most benefit with degree of pre-treatment sperm concentration not affecting magnitude of improvement across all outcomes. Lastly, we provide threshold values for $\mathrm{LH}$ and FSH where patients may no longer experience benefit from taking CC. Physicians would do well to include these data for estimating magnitude of improvement across important fertility outcomes and have better understanding the ranges of gonadotropins where patients experience the most benefit using CC.

\section{Acknowledgments}

Funding: None.

\section{Footnote}

Reporting Checklist: The authors have completed the STROBE reporting checklist. Available at https://tau. amegroups.com/article/view/10.21037/tau-21-987/rc

Data Sharing Statement: Available at https://tau.amegroups. com/article/view/10.21037/tau-21-987/dss

Peer Review File: Available at https://tau.amegroups.com/ article/view/10.21037/tau-21-987/prf

Conflicts of Interest: All authors have completed the ICMJE uniform disclosure form (available at https://tau.amegroups. com/article/view/10.21037/tau-21-987/coif). KVR and JNM are consultants for Antares Pharma, Boston Scientific, and Endo Pharmaceuticals. The other authors have no conflicts of interest to declare.

Ethical Statement: The authors are accountable for all aspects of the work in ensuring that questions related to the accuracy or integrity of any part of the work are appropriately investigated and resolved. The study was conducted in accordance with the Declaration of Helsinki (as revised in 2013). The study was approved by institutional review board of the University of California, Los Angeles (IRB \#20-000710). Prospective consenting of patients was exempt as this was a retrospective study.

Open Access Statement: This is an Open Access article distributed in accordance with the Creative Commons Attribution-NonCommercial-NoDerivs 4.0 International License (CC BY-NC-ND 4.0), which permits the noncommercial replication and distribution of the article with the strict proviso that no changes or edits are made and the original work is properly cited (including links to both the formal publication through the relevant DOI and the license). See: https://creativecommons.org/licenses/by-nc-nd/4.0/.

\section{References}

1. Thonneau P, Marchand S, Tallec A, et al. Incidence 
and main causes of infertility in a resident population $(1,850,000)$ of three French regions (1988-1989). Hum Reprod 1991;6:811-6.

2. Pan MM, Hockenberry MS, Kirby EW, et al. Male infertility diagnosis and treatment in the era of in vitro fertilization and intracytoplasmic sperm injection. Med Clin North Am 2018;102:337-47.

3. Wheeler KM, Sharma D, Kavoussi PK, et al. Clomiphene citrate for the treatment of hypogonadism. Sex Med Rev 2019;7:272-6.

4. Ko EY, Siddiqi K, Brannigan RE, et al. Empirical medical therapy for idiopathic male infertility: a survey of the American Urological Association. J Urol 2012;187:973-8.

5. Schlegel PN, Sigman M, Collura B, et al. Diagnosis and treatment of infertility in men: AUA/ASRM guideline part I. Fertil Steril 2021;115:54-61.

6. Schlegel PN, Sigman M, Collura B, et al. Diagnosis and treatment of infertility in men: AUA/ASRM guideline part II. J Urol 2021;205:44-51.

7. A double-blind trial of clomiphene citrate for the treatment of idiopathic male infertility. World Health Organization. Int J Androl 1992;15:299-307.

8. Mićić S, Dotlić R. Evaluation of sperm parameters in clinical trial with clomiphene citrate of oligospermic men. J Urol 1985;133:221-2.

9. Rŏnnberg L. The effect of clomiphene treatment on different sperm parameters in men with idiopathic oligozoospermia. Andrologia 1980;12:261-5.

10. Palmsten K, Flores KF, Chambers CD, et al. Most frequently reported prescription medications and supplements in couples planning pregnancy: the LIFE study. Reprod Sci 2018;25:94-101.

11. Jungwirth A, Giwercman A, Tournaye H, et al. European Association of Urology guidelines on male infertility: the 2012 update. Eur Urol 2012;62:324-32.

12. Padubidri VG, Daftary SN. editor(s). Shaw's textbook of gynecology. 16 ed. Amsterdam: Elsevier, 2014.

13. Trinquart L, Jacot J, Conner SC, et al. Comparison of treatment effects measured by the hazard ratio and by the ratio of restricted mean survival times in oncology randomized controlled trials. J Clin Oncol 2016;34:1813-9.

14. Katz DJ, Nabulsi O, Tal R, et al. Outcomes of clomiphene citrate treatment in young hypogonadal men. BJU Int 2012;110:573-8.
15. Taylor F, Levine L. Clomiphene citrate and testosterone gel replacement therapy for male hypogonadism: efficacy and treatment cost. J Sex Med 2010;7:269-76.

16. Ramasamy R, Scovell JM, Kovac JR, et al. Testosterone supplementation versus clomiphene citrate for hypogonadism: an age matched comparison of satisfaction and efficacy. J Urol 2014;192:875-9.

17. Moskovic DJ, Katz DJ, Akhavan A, et al. Clomiphene citrate is safe and effective for long-term management of hypogonadism. BJU Int 2012;110:1524-8.

18. Bendre SV, Murray PJ, Basaria S. Clomiphene citrate effectively increases testosterone in obese, young, hypogonadal men. Reprod Syst Sex Disord 2015.

19. Krzastek SC, Sharma D, Abdullah N, et al. Long-term safety and efficacy of clomiphene citrate for the treatment of hypogonadism. J Urol 2019;202:1029-35.

20. Alder NJ, Keihani S, Stoddard GJ, et al. Combination therapy with clomiphene citrate and anastrozole is a safe and effective alternative for hypoandrogenic subfertile men. BJU Int 2018;122:688-94.

21. Hussein A, Ozgok Y, Ross L, et al. Clomiphene administration for cases of nonobstructive azoospermia: a multicenter study. J Androl 2005;26:787-91; discussion 792-3.

22. Reifsnyder JE, Ramasamy R, Husseini J, et al. Role of optimizing testosterone before microdissection testicular sperm extraction in men with nonobstructive azoospermia. J Urol 2012;188:532-6.

23. Cannarella R, Condorelli RA, Mongioì LM, et al. Effects of the selective estrogen receptor modulators for the treatment of male infertility: a systematic review and meta-analysis. Expert Opin Pharmacother 2019;20:1517-25.

24. Keihani S, Wright LN, Alder NJ, et al. Baseline gonadotropin levels and testosterone response in hypogonadal men treated with clomiphene citrate. Urology 2020;142:119-24.

25. Sharma D, Zillioux J, Khourdaji I, et al. Improvements in semen parameters in men treated with clomiphene citrate-A retrospective analysis. Andrologia 2019;51:e13257.

26. Hashimoto T, Miyai K, Matsumoto K, et al. LH and FSH response to synthetic LHRH after consecutive administration of clomiphene citrate in normal males. J Clin Endocrinol Metab 1975;41:1110-2.

27. Whitten SJ, Nangia AK, Kolettis PN. Select patients 
with hypogonadotropic hypogonadism may respond to treatment with clomiphene citrate. Fertil Steril 2006;86:1664-8.

28. Gundewar T, Kuchakulla M, Ramasamy R. A paradoxical

Cite this article as: Jiang T, Osadchiy V, Santamaria A, Zheng MH, Modiri N, Sigalos JT, Regets KV, Mills JN, Eleswarapu SV. Initial gonadotropin levels and sperm parameters differentiate the response to clomiphene citrate in subfertile men. Transl Androl Urol 2022;11(2):116-123. doi: 10.21037/ tau-21-987 decline in semen parameters in men treated with clomiphene citrate: A systematic review. Andrologia 2021;53:e13848. 